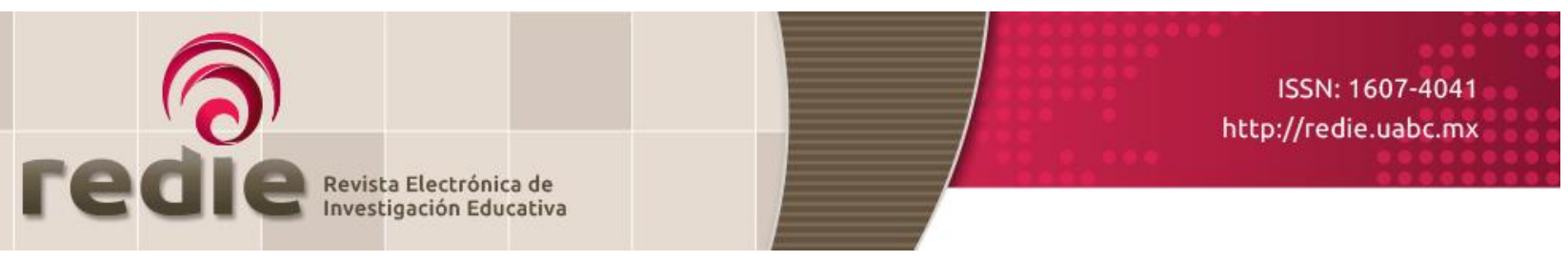

Vol. 20, Núm. 4, 2018

\title{
Estereotipos de género en Educación Física, una revisión centrada en el alumnado
}

\section{Gender Stereotypes in Physical Education: A Student-Centered Review}

\author{
Myriam Alvariñas-Villaverde (*) myalva@uvigo.es \\ Macarena Pazos-González (*) maka20111990@hotmail.es
}

(*) Universidad de Vigo

(Recibido: 29 de marzo de 2017; Aceptado para su publicación: 3 de mayo de 2017)

Cómo citar: Alvariñas-Villaverde, M. y Pazos-González, M. (2018). Estereotipos de género en Educación Física, una revisión centrada en el alumnado. Revista Electrónica de Investigación Educativa, 20(4), 154-163. https://doi.ora/10.24320/redie.2018.20.4.1840

\section{Resumen}

Este trabajo se centró en una revisión sobre estereotipos de género en Educación Física, poniendo el foco de atención en el colectivo de estudiantes. Se llevó a cabo una búsqueda en Web of Science, Scopus, SportDiscus, ERIC y Psylnfo, seleccionando artículos publicados entre 2005 y 2015. La muestra final fue de 25 documentos. Los datos revelaron la existencia de cuatro puntos de interés: el avance y resistencia al cambio de algunas creencias; la importancia de los estigmas y su repercusión en el comportamiento; la relación directa con contenidos y rendimiento; y, la figura del profesorado como agente reproductor. Se constató cierta evolución en algunos elementos, pero un avance general lento que hace urgente la puesta en práctica de alternativas de cambio profundo que aborden minuciosamente estos temas, en las que la perspectiva de género sea un eje determinante.

Palabras clave: Coeducación, deporte, roles de género, estereotipo de género.

\section{Abstract}

This work is centered on a review of gender stereotypes in physical education and focuses on students. A search was carried out on the Web of Science, Scopus, SportDiscus, ERIC, and Psylnfo, and 25 papers published between 2005 and 2015 were selected. The data reveals four centers of interest: the progress and resistence to change of certain beliefs, the importance of stigmas and their impact on behavior, the direct relationship to content and performance, and the figure of teachers as reproductive agents. Some development is noted in certain aspects, but general progress is slow, making it imperative to implement alternatives for change that address these issues and include gender perspective as a key bedrock. 


\section{Introducción}

A través del proceso de socialización se asimilan modelos o referentes simbólicos que condicionan la vida personal y social; se aprende, por ejemplo, el modelo ideal dominante de lo que debe ser un hombre o una mujer y las funciones que debe y puede cumplir (Fernández-Valencia, 2004). Así, se interiorizan ideas preconcebidas, representaciones generalizadas sobre los comportamientos que hombres y mujeres deben mostrar; son los estereotipos de género, atribuciones culturales cambiantes en diferentes espacios y tiempos.

Diversos trabajos han demostrado la influencia social de estereotipos de género que proponen una lógica de cuidado y afecto en la construcción social de la feminidad y una lógica de dominio, competición y control en la construcción social de la masculinidad (Rebollo-Catalán, Ruiz-Pinto y García-Pérez, 2017). La familia, el grupo de iguales, los medios de comunicación y la escuela, como principales agentes socializadores, son los encargados de transmitir dichas ideas estereotipadas. Dentro del contexto escolar, las clases de Educación Física suponen un espacio en el que las diferencias entre representaciones de género se hacen más notables.

En los últimos tiempos los papeles de hombres y mujeres dentro de la sociedad han ido variando (Sicilia, 2003). Además, las políticas de igualdad que se van implantando de forma paulatina promueven el principio de equidad de género, de manera que existe la obligación de educar en igualdad. Pese a ello, no se termina de cumplir este objetivo en las aulas de Educación Física (Sicilia, 2003). Valdivia, Sánchez-Pato, Alonso y Zágalaz (2010) señalan que se ha avanzado mucho en materia de coeducación, pero aún no se ha conseguido todo lo que se suponía. Indican, por ejemplo, que se debe asegurar un trato equitativo mediante la superación de estereotipos de género y el rechazo del modelo universal androcéntrico, desarrollando las cualidades de cada individuo.

Las alumnas han tenido que asumir un currículum esencialmente masculino, el cual es fomentado por los docentes. En general, los alumnos disfrutan de programas y contenidos más acordes a sus necesidades, lo que provoca en ocasiones que las mujeres tengan una experiencia insatisfactoria en las clases (Baena y Ruiz, 2009; Blández, Fernández-García y Sierra, 2007). Además, las vivencias negativas afectan también a aquellos varones que se alejan del modelo de masculinidad hegemónica (Vidiella, 2007). Aun así, destaca cierto avance respecto a la representación de los estereotipos más tradicionales relacionados con la materia pues, si bien todavía hay creencias que se mantienen, son menos los sujetos que las reafirman, mostrando una clara resistencia ante ese modelo masculino dominante (Fernández-García, 2007).

La lucha contra estereotipos de género en un ámbito tan específico como el de la Educación Física requiere de un importante seguimiento de investigación, a fin de conocer de forma más profunda si se van logrando las metas establecidas y verificar qué métodos y buenas prácticas son eficaces en relación con este tema. ¿Qué falsos mitos han quedado atrás? ¿Qué creencias se siguen manteniendo y se resisten al cambio? ¿Cómo influyen los estereotipos en la asignatura? ¿Existen formas de combatirlos? ¿Se llevan a cabo intervenciones que partan de principios como beneficiar la percepción de competencia y diversificar los contenidos? ¿Qué papel juega la figura docente? En definitiva, ¿cuáles son las evidencias actuales y qué informaciones se asocian a este concepto?

Рara dar respuesta a estas y otras cuestiones, este trabajo se centra en conocer el estado actual de la cuestión a través del análisis del material publicado en los últimos años. Se ha realizado una revisión exhaustiva de esta temática, por un lado, porque es necesario entender que es de gran interés para la formación y, por otro, para apoyar el estudio de este conocimiento específico dentro del contexto de la Educación Física, en el que todavía queda mucho por hacer. El objetivo de la investigación fue analizar los artículos vinculados a los estereotipos de género en Educación Física, prestando especial interés al colectivo de estudiantes. 


\section{Método}

En el estudio se siguió un diseño descriptivo a partir del análisis de los documentos seleccionados. El proceso se realizó tomando como referencia las recomendaciones descritas por Perestelo-Pérez (2013) para la realización de revisiones sistemáticas. Las bases de datos empleadas como fuentes de información fueron Web of Science, Scopus, SportDiscus, ERIC y Psylnfo. La ecuación utilizada fue: ("gender stereotyp*" OR "sex stereotyp*") AND "physical education" AND ("student OR adolescen*" OR "child*" OR "school"). Para refinar la búsqueda se estableció como criterio de inclusión que los documentos pertenecieran al período comprendido entre 2005 y 2015.

En una primera exploración se obtuvieron 95 documentos. Se excluyeron los estudios de revisión sistemática, reseñas, tesis doctorales y capítulos de libro, de forma que el corpus de trabajo se concentró en artículos de revistas científicas. Asimismo, se descartaron los trabajos escritos en idiomas diferentes al castellano, portugués, francés o inglés. Tampoco se seleccionaron estudios que, aun teniendo relación con el tema, se concentraban en cuestiones muy particulares que se alejaban del objeto de estudio o que se centraban específicamente en la visión del profesorado.

Después de descartar duplicados y aplicar los criterios de exclusión, los resultados válidos para el estudio fueron 14. Las referencias de los estudios seleccionados fueron rastreadas con la intención de localizar artículos que no hubieran aparecido en la búsqueda inicial. A partir de esto se añadieron 11 referencias más que abordaban el tema. Por tanto, la muestra final fue de 25 artículos.

Todo el proceso fue realizado por dos investigadoras y supervisado por un tercero que se encargó de repasar el método de búsqueda, así como de revisar el material excluido de la muestra, corroborando que los criterios aplicados eran coherentes con las finalidades del estudio. El análisis de la información se llevó a cabo de forma inductiva. La figura 1 muestra el diagrama de flujo de la revisión.

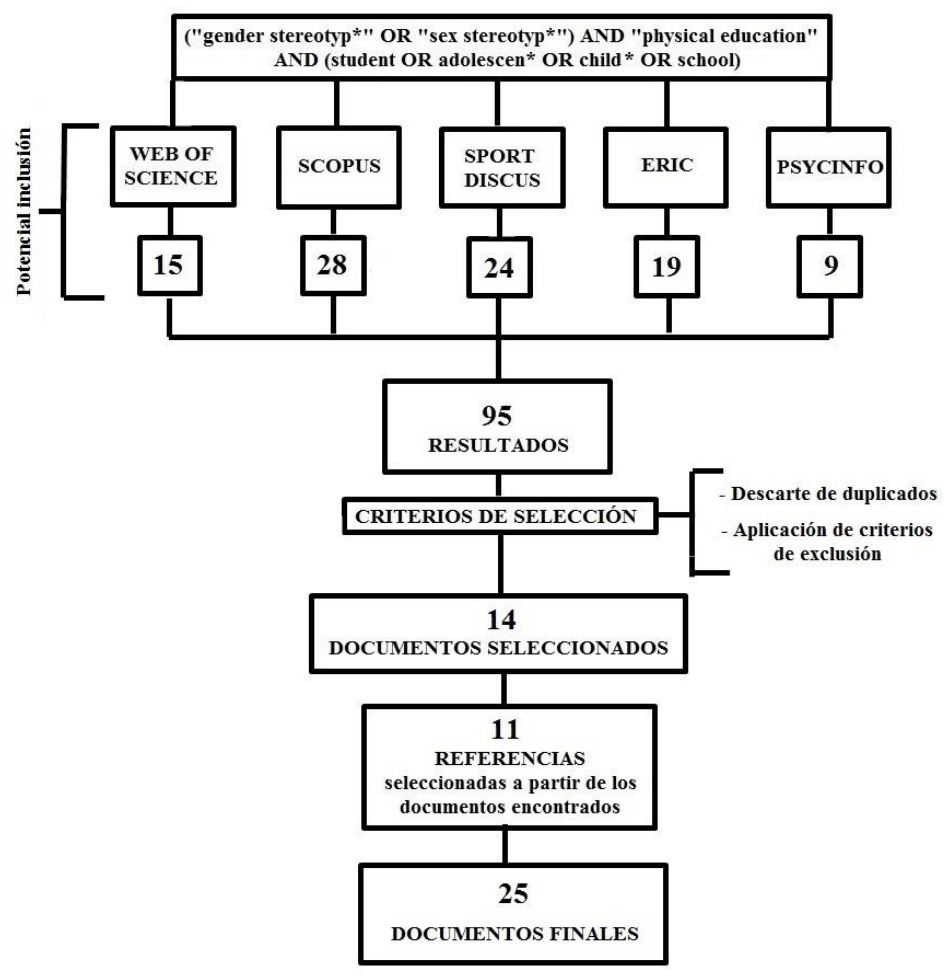

Figura 1. Diagrama de flujo de la revisión 


\section{Análisis de los datos}

La mayor parte de los trabajos se hicieron en Francia y España; y en menor proporción se encontraron otros procedentes de Estados Unidos, Rumania, Brasil, Canadá, Dinamarca, Grecia, Croacia y Turquía.

El alumnado de educación secundaria fue el colectivo más estudiado, seguido del de primaria y el universitario. La mayoría de muestras seleccionadas corresponde a discentes; sólo cuatro estudios presentaron muestras de docentes y estudiantes. Se utilizaron diferentes instrumentos, entre los que destacan los cuestionarios, las escalas y la observación directa; otros menos utilizados fueron las entrevistas, los grupos de discusión y los diarios de campo.

Si se analiza el tipo de revistas en las que se publicaron los materiales, se observa una predominancia de las relativas al contexto de la Educación Física y del Deporte, aunque también destacan las de ámbito psicológico y educativo.

El análisis de resultados mostró que algunas investigaciones estudiaban directamente los estereotipos, mientras que otras se relacionaban con ellos de forma menos directa. Así, se encontraron documentos cuyo objetivo era, por ejemplo, examinar las relaciones entre estereotipos de género y actividad física o analizar qué estereotipos siguen vigentes en la actualidad. Por otra parte, algunos trabajos se centraban en cuestiones como examinar la efectividad de un programa para combatir el sexismo o las diferencias de actitud ante contenidos considerados masculinos o femeninos.

Ideas como que hacer mucho deporte resta feminidad a las mujeres o que los varones que hacen danza son menos masculinos se rechazan de forma mayoritaria (Alvariñas, Fernández-Villarino y López-Villar, 2009). Zaravigka y Pantazis (2012) constatan también una propensión al rechazo de ideas como que la mujer que es buena en el deporte sea un "marimacho" y la aceptación de que la Educación Física está en la naturaleza femenina. Estas tendencias indican una clara evolución respecto a trabajos realizados años atrás, que indicaban la permanencia de opiniones como que el deporte masculiniza a las mujeres (Instituto de la Mujer, 2006); se enfrentan, además, a antiguos falsos mitos relacionados con prejuicios de tipo sexual o argumentos de riesgo para la reproducción de la mujer, entre otros (Macías, 1999).

No obstante, el análisis de los artículos mostró la resistencia al cambio del estereotipo que afirma que existen unos deportes más apropiados para hombres y otros más apropiados para mujeres. Blández et al. (2007) indican que tanto el alumnado de primaria como el de secundaria perciben que determinadas actividades físico-deportivas tienen marca de género, reproduciendo en sus discursos las tipificaciones más tradicionales. Lo mismo sucede en los trabajos de Hannon, Soohoo, Reel y Ratliffe (2009) o Schmalz, Kerstetter y Anderson (2008).

Se observó, asimismo, un discurso sobre el juego en el recreo fundamentado en la creencia de que existen deportes diferenciados para hombres y mujeres definidos por las competencias corporales que requieren (Pawlowski, Ergler, Tjørnhøj-Thomsen, Schipperijn y Troelsen, 2015). Además, estas categorizaciones están asociadas a otros aspectos, como la construcción del cuerpo y las tendencias de imagen corporal para unas y otros (González, Ruiz y Carrasco, 2007).

En una investigación reciente, Alvariñas y Novoa (2016) observan que la mayoría de estudiantes cree que las chicas pueden hacer cualquier tipo de deporte. También se considera que el tipo de práctica físicodeportiva que se haga no depende de si eres chico o chica; por ello hablan de un avance o evolución en este sentido. Algo similar ocurre en el trabajo de Zaravigka y Pantazis (2012), ya que los chicos consideran que las niñas están al mismo nivel que ellos en lo que al deporte se refiere. Sin embargo, cuando se propone que los deportes son más o menos adecuados en función de si eres hombre o mujer, en el primer estudio la mayoría de los estudiantes está de acuerdo con esta idea, y en el segundo, pese a la tendencia al rechazo, se observa que un porcentaje importante aún lo considera así. 


\subsection{Estigmas y miedos con marca de género}

La revisión desveló que el miedo al estigma o desaprobación social puede influir en la conducta del alumnado, actuando incluso como predictor de participación en la niñez (Schmalz et al., 2008). El hecho de participar en actividades deportivas consideradas poco apropiadas para una persona conlleva el riesgo de ser juzgada y etiquetada por la sociedad (Chalabaev, Sarrazin y Fontayne, 2009; Laurin, 2013). Por ello, algunos varones rechazan la danza y evitan la realización de ciertos movimientos, por miedo a perder su masculinidad (Kleinubing, Saraiva y Francischi, 2013) o manifiestan conductas de huida ante contenidos considerados femeninos (Koca, 2009), mientras las chicas abandonan las actividades de perfil más masculino (Koca, 2009).

Tal y como demuestra Laurin (2013), la presencia de creencias estereotipadas produce ansiedad en las personas afectadas, facilitando la interacción entre estudiantes que se unen ante la amenaza del estereotipo. En la misma línea, Chalabaev, Dematte, Sarrazin y Fontayne (2014) observan que la eliminación de las conductas estereotipadas contribuye a aliviar los efectos de esta amenaza.

Pero, ¿están igualmente expuestos a estos estigmas hombres y mujeres? Pareciera que afectan al alumnado en general, pero estudios como el de Schmalz y Kerstetter (2006) y Schmalz et al. (2008) destacan la menor presión de las niñas para ejercitarse en disciplinas tipificadas como masculinas. Incluso se observa que no existe relación entre la conciencia de estigma y el tipo de participación (competitiva/recreativa) en las niñas, pero sí en los niños: los que participan en deportes considerados masculinos tienen mayor conciencia de estigma cuando lo hacen de forma recreativa que cuando lo hacen de forma competitiva.

Las chicas perciben como neutras más disciplinas (Fernández-García, 2007) y se alejan en mayor medida de concepciones estereotipadas (Alvariñas y Novoa, 2016; Zaravigka y Pantazis, 2012). Por su parte, Pelegrín, León, Ortega y Garcés (2012) indican que los niños tienen más actitudes sexistas que las niñas, tanto antes como después de poner en marcha un programa de intervención. Además, las niñas practican muchas disciplinas que tradicionalmente se atribuían a los hombres, mientras que la situación inversa no se da con la misma intensidad (Blández et al., 2007; Gracia-Marco et al., 2010; Schmalz et al., 2008).

Por otra parte, las mujeres también sufren dificultades en el ámbito de la educación corporal. En algunos casos, las alumnas obtienen y esperan peores notas en Educación Física, mientras que los varones catalogados como andróginos o masculinos obtienen calificaciones más altas (Lentillon, Cogérino y Kaestner, 2006). Asimismo, les cuesta más involucrarse en los juegos en los que también participan varones (Petracovschi, Bosioc, Voicu y Tabärä-Amanar, 2011) o incluso no quieren participar debido al mal comportamiento de sus compañeros, que incluye comentarios sexistas (Koca, 2009). Además, son ignoradas cuando no dominan las técnicas (Duarte y Mourão, 2007) y ocupan espacios que potencian menos la práctica (Fagrell, Larsson y Redelius, 2012, Pawlowski et al., 2015).

\subsection{Contenidos y rendimiento. Efectos de las propuestas coeducativas}

La revisión describe la importancia de la percepción de competencia en el rendimiento y que ésta se relaciona directamente con el contenido impartido. Chalabaev y Sarrazin (2009) constatan el vínculo entre estereotipos de género y motivación hacia la actividad física, de forma que el alumnado elige actividades que le aportan mayor percepción de competencia.

Las chicas se ven a sí mismas como inferiores en deportes asociados a lo masculino, como es el caso del futbol (Petracovschi, Voicu, Faur y Sinitean-Singer, 2011). El estereotipo de que las chicas son peores en futbol predice negativamente la percepción de habilidad y ésta, a su vez, el rendimiento. Asimismo, el rol de masculinidad predice la creencia sobre el peor rendimiento de las chicas, la percepción de habilidad y la creencia de que los chicos son mejores en futbol (Chalabaev, Sarrazin y Fontayne, 2009).

En esta línea argumentativa, y teniendo en cuenta que la habilidad es un factor de influencia en la exclusión de las chicas (Chan-Vianna, Luz y Mourão, 2010), algunos trabajos apuntan que a las mujeres les 
gustaría tener contenidos diferentes en sus clases, que les permitieran ampliar su bagaje motriz y que se hiciera desde una perspectiva menos competitiva (Duarte y Mourão, 2007; Woodson-Smith, Dorwart y Linder, 2015).

En la revisión hecha, investigaciones como las de Kleinubing et al. (2013), Vlašic, Oreb y Katobic (2012) y Chalabaev, Sarrazin, Trouilloud y Jussim (2009) estudian las actitudes a partir de la puesta en práctica de la danza o de la gimnasia en el aula -disciplinas ligadas tradicionalmente más al mundo femenino que al masculino, en las que la percepción de competencia en las chicas es mayor. Cuando se desarrollan estos contenidos, las chicas tienen actitudes más positivas y obtienen mejores resultados. Asimismo, trabajos como el de Vlašic et al. (2012) demuestran que a partir de la vivencia de sesiones de danza los chicos mejoran notablemente su actitud y se muestran favorables hacia la misma.

Las intervenciones de Drouin, Varga y Gammage (2008) son un buen ejemplo de que cuando el alumnado rompe el miedo o supera la vergüenza inicial que le provoca enfrentarse a dichas prácticas, experimenta, en la mayor parte de los casos, sensaciones positivas. En este caso se demuestra la inexistencia de diferencias de rendimiento en función del género.

En la educación primaria, Pelegrín et al. (2012) comprueban la efectividad de su programa y revelan que éste influye positivamente en la disminución de actitudes sexistas tanto en niños como en niñas. Además, se demuestra que el trabajo en parejas mixtas puede mejorar la cooperación y la comunicación entre chicos y chicas, fomentando un mejor clima en el aula (Petracovschi, Bosioc et al., 2011). También el rendimiento de las niñas puede aumentar ante la presencia de discursos motivacionales (Chalabaev et al., 2014).

Sin embargo, no se observan resultados parecidos en el trabajo de Petracovschi, Voicu et al. (2011). Después de la intervención se aprecian ligeros cambios de actitud en la aceptación que los chicos tienen de las chicas, y viceversa; pero globalmente los cambios no son extraordinarios. Se concluye en todo caso que el trabajo con grupos mixtos contribuye a la activación de las chicas en este campo y, con ello, al cambio en el pensamiento de los varones sobre sus capacidades.

Por otra parte, hay que destacar apreciaciones como las de Clement-Guillotin, Cambon et al. (2013) en el sentido de que el alumnado puede realizar actividades asociadas a un sexo diferente al suyo únicamente por obtener la aprobación de sus docentes. Es importante, por tanto, que el cambio de conducta proceda de motivaciones intrínsecas.

No obstante, y a pesar de estas evidencias excepcionales, en el análisis realizado se observó que cuando se plantean propuestas de intervención alternativas para favorecer la equidad, los resultados son mayoritariamente positivos y bastante valiosos desde el punto de vista pedagógico, y los resultados son acordes a los obtenidos por Kleinubing et al. (2013) cuando argumentan que el uso de este tipo de prácticas puede suponer una importante herramienta para romper estereotipos ligados a ciertas disciplinas.

\subsection{El personal docente, ¿reproductor de estereotipos o agente de cambio?}

El análisis de los artículos permitió comprobar cómo percibe el estudiantado algunas cuestiones relativas al profesorado, encontrándose ciertas diferencias en función de los artículos.

Por una parte, se cuestiona el rol que ejerce en su práctica diaria, pudiendo ser un importante reproductor de creencias estereotipadas. Algunos estudios destacan que el estudiantado percibe un trato diferenciado de forma que se es más permisivo (Pawlowski et al., 2015) o más tolerante con las alumnas y más exigente con los alumnos (Koca, 2009). Asimismo, se interactúa más con ellos en las clases y se utilizan discursos que contribuyen a reforzar estereotipos de género (Кoca, 2009).

También, cuando hay que seleccionar a estudiantes para hacer equipos, algunas alumnas perciben que las profesoras eligen primero a las más habilidosas (Duarte y Mourão, 2007) y en el ámbito universitario se 
constata que tanto el profesorado como el alumnado eligen a más hombres que mujeres. Incluso se elogia más a las estudiantes por su desempeño y se asocia su práctica con la aceptación social, mientras que la participación de los varones está vinculada a la probabilidad de tener éxito (Clement-Guillotin, Chalabaev y Fontayne, 2013). Se percibe también que el acompañamiento que se hace está ligado íntimamente a si se es chico o chica, de forma que las profesoras están más con las alumnas y los profesores más con los alumnos (Pawlowski et al., 2015).

En relación con las expectativas, Chalabaev, Sarrazin et al. (2009) comprueban que los estereotipos favorables a los alumnos pueden influenciar las expectativas del profesorado respecto a su rendimiento y esto influye aun cuando no existen diferencias de rendimiento entre hombres y mujeres, e incluso cuando las ejecuciones de las niñas son mejores.

Por otra parte, se encontraron trabajos en los que globalmente se valora como neutra o exenta de prejuicios la figura docente y, sin embargo, ciertas valoraciones dejan la puerta abierta para el debate. Por ejemplo, Zaravigka y Pantazis (2012) determinan que el alumnado en general manifiesta tendencia a percibir una actitud neutral por parte del cuerpo docente. Sin embargo, las alumnas consideran que no tienen las mismas oportunidades de acondicionamiento físico en las clases y gran parte del alumnado piensa que no se propone a los chicos realizar actividades consideradas femeninas.

De igual forma, en el estudio de Duarte y Mourão (2007) muchas alumnas perciben que el profesorado garantiza la igualdad de oportunidades en las clases, aunque echan en falta que los contenidos sean más diversos, más cooperativos y les permitan ampliar sus experiencias motrices. Ambos estudios resaltan, además, que se pone demasiado énfasis en la técnica (Duarte y Mourão, 2007) o en la fuerza y la velocidad (Zaravigka y Pantazis, 2012), a partir de un patrón culturalmente definido por los varones. Esto sugiere que en estas investigaciones desde la perspectiva general del alumnado se aprecia una actitud docente positiva y con tendencia a la imparcialidad, pero que en la práctica diaria existen matices que hacen que dicha imparcialidad no sea tan absoluta si se hace un análisis más profundo.

\section{Conclusiones}

La realización de este trabajo permitió conocer las temáticas que se están estudiando en los últimos años relacionadas con los estereotipos en el campo de la Educación Física:

En primer lugar, se aprecia que se han superado antiguas creencias y que se están dando matices de cambio ante un estereotipo muy arraigado que afirma que hay deportes que son para mujeres y otros para hombres. Pese a la resistencia al cambio, el alumnado se sitúa en un panorama más neutro de pensamiento y de acción.

En segundo lugar, se observa que el alumnado convive en realidades donde el género tiene una importancia fundamental. Esto afecta tanto a varones como a mujeres, produciendo sentimientos de vergüenza, ansiedad, miedo o incapacidad y comportamientos derivados poco adaptativos. Es fundamental elegir cuáles contenidos y qué estrategias facilitadoras deben plantearse para abordar la diversidad de género, de manera que haya un equilibrio y que las diferencias no supongan un problema sino una oportunidad para aprender de la pluralidad de masculinidades y feminidades que existen.

En tercer lugar, se comprobó que las actividades físicas y deportivas planteadas en el aula pueden constituir una herramienta de perpetuación de estereotipos o de ruptura de los mismos. La clave, desde nuestro punto de vista, está en cómo se abordan, en la metodología y recursos empleados.

En cuarto lugar, la figura docente puede activar un proceso de buenas prácticas de lucha contra los estereotipos, promoviendo cambios de actitud, o bien ser agente reproductor de diferencias. Una parte del corpus de artículos aportó experiencias prácticas alternativas para deconstruir prejuicios ligados tanto al mundo femenino como al masculino. No obstante, parece que en lo que respecta a los y las profesionales hay muchos elementos que necesitan ser mejorados en la práctica diaria. 
Como límites del estudio resalta la comparación de resultados a partir de instrumentos y muestras diferentes, así como la diferencia que supone el contrastar datos de países de culturas y contextos específicos, así como el no caracterizar los marcos metodológicos de las investigaciones.

Una línea de investigación futura implicaría realizar intervenciones con perspectiva de género. Esto supondría un planteamiento que englobe todos los elementos didácticos, comenzando por las competencias y los objetivos que se persiguen; continuaría con una cuidadosa conciliación de contenidos y especificidad del trabajo metodológico y finalizaría con fórmulas de evaluación equilibradas. El interés residirá en comprobar la efectividad de diferentes programas y en las consecuencias generadas tanto en discentes como en docentes.

Por otro lado, se detectó que tanto en el ámbito universitario como en las primeras etapas los estudios son menos abundantes. Se sugiere, por tanto, más investigaciones en estas franjas educativas.

Otra línea en la que se hace necesario ahondar es la relativa a la formación del profesorado, de forma que se pueda luchar contra el currículo oculto, analizar en qué aspectos se comenten errores y mostrar qué estrategias son útiles para abordar una educación equitativa en clave de género. Esta línea incluye también al profesorado en preservicio, aportándoles recursos para que cuando terminen sus estudios cuenten con los conocimientos y sensibilidad adecuados para hacer ajustes eficaces en esta materia.

\section{Referencias}

Alvariñas, M. y Novoa, A. (2016). Pensamientos relacionados con la actividad física y el género en adolescentes de Galicia. Sportis, 2(1), 23-35. doi:10.17979/sportis.2016.2.1

Alvariñas, M., Fernández-Villarino, M. A. y López-Villar, C. (2009). Actividad física y percepciones sobre deporte y género. Revista de Investigación en Educación, 6, 113-122. Recuperado de http://webs.uvigo.es/reined/

Baena, A. y Ruiz, P. J. (2009). Tratamiento educativo de la coeducación y la igualdad de sexos en el contexto escolar y en especial en educación física. Aula Abierta, 37(2), 111-122.

Blández, J., Fernández-García, E. y Sierra, M. A. (2007). Estereotipos de género, actividad física y escuela: La perspectiva del alumnado. Profesorado. Revista de Currículum y Formación del Profesorado, 11(2), 1-21.

Chalabaev, A. y Sarrazin, P. (2009). Relationship between sex stereotypes related to sports and students' self-determined motivation in physical education classes. Science et Motricite, 66(1), 61-70.

doi:10.3917/sm.066.0061

Chalabaev, A., Sarrazin, P. y Fontayne, P. (2009). Stereotype endorsement and perceived ability as mediators of the girls gender orientation-soccer performance relationship. Psychology of Sport and Exercise, 10(2), 297-299. doi:10.1016/j.psychsport.2008.08.002

Chalabaev, A., Sarrazin, P., Trouilloud, D. y Jussim, L. (2009). Can sex-undifferentiated teacher expectations mask an influence of sex stereotypes? Alternative forms of sex bias in teacher expectations. Journal of Applied Social Psychology, 39(10), 2469-2498. doi:10.1111/j.1559-1816.2009.00534.x

Chalabaev, A., Dematte, E., Sarrazin, P. y Fontayne, P. (2014). Creating regulatory fit under streotype threat: Effects on performance and self-determination among junior high school students. Revue Internationale de Psychologie Sociale, 27(3-4), 119-132.

Chan-Vianna, A., Luz, D. y Mourão, L. (2010). Educação Física, gênero e escola: Uma análise da produção acadêmica [Educación Física, género y escuela: Un análisis de la producción académica]. Movimento, 16(2), $149-166$ 
Clement-Guillotin, C., Chalabaev, A. y Fontayne, P. (2013). Disentangling the influences of sex stereotypes in physical education with the non-zero-sum/zero-sum behaviors distinction. Movement \& Sport Sciences/Science \& Motricité, 79, 55-61. doi:10.1051/sm/2012029

Clement-Guillotin, C., Cambon, L., Chalabaev, A., Radel, R., Michel, S. y Fontayne, P. (2013). Social value and asymmetry of gender and sex categories in physical education. Revue Europeene de Psychologie Appliquee, 63(2), 75-85. doi:10.1016/j.erap.2012.12.004

Drouin, B., Varga, H. y Gammage, K. L. (2008). The positive exerciser stereotype: the role of gender stereotype of the activity. Journal of Applied Biobehavioral Research, 13(3), 143-156.

doi:10.1111/j.1751-9861.2008.00032.x

Duarte, C. P. y Mourão, L. (2007). Representações de adolescentes femininas sobre os critérios de seleção utilizados para a participação em aulas mistas de educação física [Representaciones de adolescentes femeninas sobre los criterios de selección utilizados para la participación en clases mixtas de educación física]. Movimento, 13(1), 37-56.

Fagrell, B., Larsson, H. y Redelius, K. (2012). The game within the game: girls' underperforming position in Physical Education. Gender and Education, 24(1), 101-118. doi:10.1080/09540253.2011.582032

Fernández-García, E. (Coord.) (2007). Memoria del estudio de los estereotipos de género vinculados con la actividad física y el deporte en los centros docentes de Educación Primaria y Secundaria. Madrid: Instituto de la Mujer.

Fernández-Valencia, A. (2004). El género como categoría de análisis en la enseñanza de las Ciencias Sociales. En I. Vera-Muñoz y D. Pérez i Pérez (Coords.), Formación de la ciudadanía: las tic y los nuevos problemas (pp. 1-24). Alicante: Asociación Universitaria de Profesores de Didáctica de las Ciencias Sociales.

González, J. M., Ruiz, L. M. y Carrasco, M. (2007). The social construction of gender in spanish physical education students. Sport, Education and Society, 12(2), 141-158.

Gracia-Marco, L., Tomàs, C., Vicente-Rodríguez, G., Jiménez-Pavón, D., Rey-López, J. P., Ortega, F. B., ... Moreno, L. A. (2010). Extra-curricular participation in sports and socio-demographic factors in spanish adolescents: The AVENA study. Journal of Sports Sciences, 28(13), 1383-1389.

doi:10.1080/02640414.2010.510846

Hannon, J., Soohoo, S., Reel, J. y Ratliffe, T. (2009). Gender stereotyping and the influence of race in sport among adolescents. Research Quarterly For Exercise and Sport, 80(3), 676-684.

Instituto de la Mujer (2006). Actitudes y prácticas deportivas de las mujeres en España (1900-2005). Madrid: Autor.

Kleinubing, N. D., Saraiva, M. C. y Francischi, V. G. (2013). Dance in high school: reflections on gender stereotypes and movement. Revista da Educaçao Física, 24(1), 71-82.

Koca, C. (2009). Gender interaction in coed physical education: a study in Turkey. Adolescence, 44(173), 165-185.

Laurin, R. (2013). Stereotype threat and lift effects in motor task performance: the mediating role of somatic and cognitive anxiety. The Journal of Social Psychology, 153(6), 687-699.

Lentillon, V., Cogérino, G. y Kaestner, M. (2006). Injustice in physical education: gender and the perception of deprivation in grades and teacher support. Social Psychology of Education, 9(3), 321-339. 
Macías, V. (1999). Estereotipos y deporte femenino. La influencia del estereotipo en la práctica deportiva de niñas y adolescentes. Tesis doctoral no publicada. Universidad de Granada. Recuperado de http://hera.ugr.es/tesisugr/15755368.pdf

Pawlowski, C. S., Ergler, C., Tjørnhøj-Thomsen, T., Schipperijn, J. y Troelsen, J. (2015). 'Like a soccer camp for boys': a qualitative exploration of gendered activity patterns in children's self-organized play during school recess. European Physical Education Review, 21(3), 275-291. doi:10.1177/1356336X14561533

Pelegrín, A., León, J. M., Ortega, E. y Garcés, E. J. (2012). Programa para el desarrollo de actitudes de igualdad de género en clases de educación física en escolares. Educación XX1, 15(2), 271-292.

Perestelo-Pérez, L. (2013). Standards on how to develop and report systematic reviews in Psychology and Health. International Journal of Clinical and Health Psychology, 13(1), 49-57.

Petracovschi, S., Bosioc, B., Voicu, S. y Tabärä-Amanar, S. (2011). Gender, competition and cooperation in scholar physical education. Movement and Health, 11(2), 228-235.

Petracovschi, S., Voicu, S., Faur, M. y Sinitean-Singer, F. (2011). Promote the equality and fairness for everyone in physical education activity-the case of mixed group. Journal of Physical Education and Sport, 11(1), 81-86.

Rebollo-Catalán, A., Ruiz-Pinto, E. y García-Pérez, R. (2017). Preferencias relacionales en la adolescencia según el género. Revista Electrónica de Investigación Educativa, 19(1), 58-72. Recuperado de http://redie.uabc.mx/redie/article/view/1022

Schmalz, D. L. y Kerstetter, D. L. (2006). Girlie girls and manly men: children's stigma consciousness of gender in sports and physical activities. Journal of Leisure Research, 38(4), 536-557.

Schmalz, D. L., Kerstetter, D. L. y Anderson, D. M. (2008). Stigma consciousness as a predictor of children's participation in recreational vs. competitive sports. Journal of Sport Behavior, 31(3), 276-297.

Sicilia, A. (2003). La investigación sobre el pensamiento del alumnado. Una revisión desde la educación física. Revista de Educación, 331, 577-613.

Valdivia, P. A., Sánchez-Pato, A., Alonso, J. I. y Zágalaz, M. L. (2010). La coeducación en el área de educación física en España: una reseña histórica. Cultura, Ciencia y Deporte, 5(14), 77-83.

Vidiella, J. (2007). El deporte y la actividad física como mediadores de modelos corporales: género y sexualidad en el aprendizaje de las masculinidades. Educación Física y Ciencia, 9, 81-101. Recuperado de http://www.memoria.fahce.unlp.edu.ar/art revistas/pr.3305/pr.3305.pdf

Vlasic, J., Oreb, G. y Katovic, D. (2012). Dance attitude differences between female and male students. Movement and Health, 12(2), 417-421.

Woodson-Smith, A., Dorwart, C. E. y Linder, A. (2015). Attitudes toward physical education of female high school students. Physical Educator, 72(3), 460-479.

Zaravigka, K. y Pantazis, V. (2012). Equality of the genders in physical education: The studends' perceptions. Journal of Physical Education and Sport, 12(3), 350-357. doi:0.7752/jpes.2012.03052 